\title{
Digital Control of Magnetic Levitation System using Fuzzy Logic Controller
}

\author{
Shekhar Yadav \\ Dept. of Electrical Engg., \\ Institute of Technology, BHU, \\ Varanasi-221005, India
}

\author{
J.P.Tiwari \\ Dept. of Electrical Engg., \\ Institute of Technology, BHU, \\ Varanasi-221005, India
}

\author{
S.K.Nagar \\ Dept. of Electrical Engg., \\ Institute of Technology, BHU, \\ Varanasi-221005, India
}

\begin{abstract}
This paper deals with the control aspects of magnetic levitation system using fuzzy logic controller (FLC). The magnetic levitation system is a mechatronic system already acknowledged and accepted by the field experts. For such a system it is desired to proposed a suitable controller for positioning a metal sphere in air space by the help of an electromagnetic force. In the ideal situation, the magnetic force produced by current from an electromagnet counteracts the weight of the metal sphere. Nevertheless, the electromagnetic force is very sensitive, and there is noise that creates acceleration forces on the metal sphere, causing the sphere to move into the unbalanced region. FLC proposed in this paper is to control the nonlinear magnetic ball suspension system using Mamdani implication method. Samples of simulation results with different initial conditions concerning the ball's position and speed are provided included to validate the theory. The effectiveness of this proposed technique is validated through experimental results obtained by performing experiments on Feedback make Magnetic levitation system (33-210)
\end{abstract}

\section{Keywords}

Magnetic levitation system, Fuzzy logic control, PID control

\section{INTRODUCTION}

The Magnetic Levitation System (Maglev) serves as a simple model of devices that have become more popular in recent years such as Maglev trains and magnetic bearings. Maglev trains are recently tested and some lines are already available for example in Shanghai. Magnetic bearings are used in turbines for the same reason as Maglev trains are being built, which is low friction in the bearing itself. Already many turbines are in commercial used where the rotating shaft is levitated with magnetic force. Some other magnetic bearing applications include pumps, fans and other rotating machines $[7,10]$.

The magnetic levitation systems are appealing for their additional possibility of active vibration damping. This can be done by implementing different control algorithms without any hardware modification to the mechanical parts of the whole system [9]. The Maglev unit allows for the design of different controllers and test in real time using Matlab and Simulink environment.

Fuzzy logic controller (FLC) is an attractive alternative to existing classical or modern controllers for designing the nonlinear control systems. It does not require system models or complex mathematical equations describing the relationship between inputs and outputs. Fuzzy rules are very easy to learn and can be implement, even by non-experts. It typically takes only a few rules to describe systems that may require several lines of conventional software codes, which reduce the design complexity $[1,3,6]$. Although PID control is a proficient technique for handling non-linear systems but modeling these systems is often troublesome and sometimes impossible using the laws of physics. Therefore, the use of a classical controller is not suitable for nonlinear control application. Alternatively, Fuzzy Logic Control well suited for processes that are too complex for analysis by conventional techniques or when the available sources of information are interpreted qualitatively, inexactly, or uncertainly.

PID control requires the model of the system for the determination of the parameters of PID controller using control theory and finally the development of an algorithm for the controller [2]. However, in case of fuzzy logic, the system behavior is characterized using human knowledge which directly deals with the design of control algorithm on the basis of fuzzy rules. These rules are described in terms of the relationship of inputs to their corresponding outputs, and precisely determine the controller parameters. Any adjustment or debugging only requires modification in these fuzzy rules instead of redesigning the controller $[4,5,8]$. Hence control technique based on fuzzy logic not only simplifies the design, but also reduces the monotonous task of solving complex mathematical equations of nonlinear systems.

\section{MODELING OF MAGNETIC LEVITATION SYSTEM}

The Magnetic levitation system as shown Fig. 1, consists of a magnetic sphere suspension system. The objective of the system is to control the vertical position of the ball by adjusting the current in the electromagnet through the input voltage $V_{i n}$. The metal sphere is suspended in air by the electromagnetic force generated by an electromagnet. 


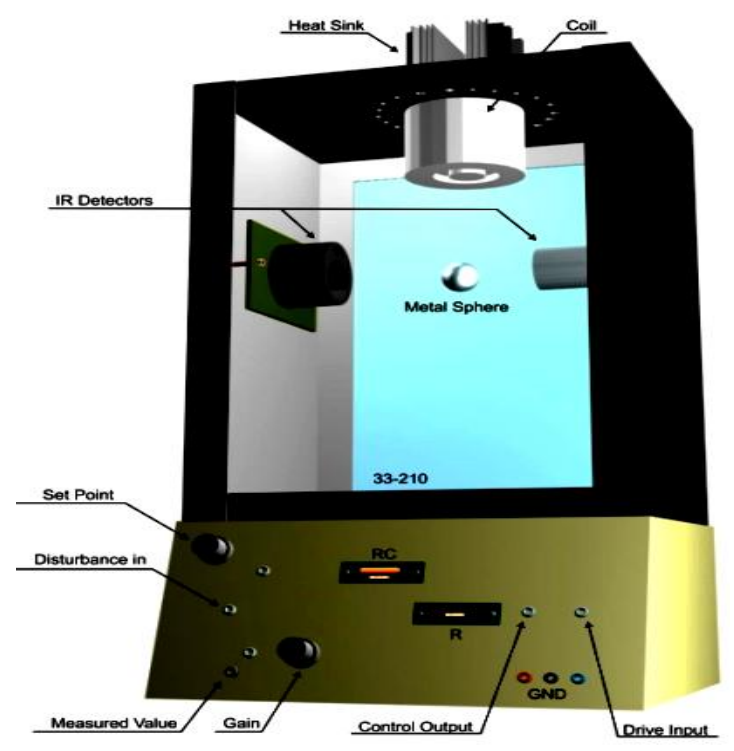

Figure 1 Maglev mechanical unit

Fig 1: Maglev Mechanical Unit

The Magnetic levitation system consists of an electromagnet, a metal sphere and an infra-red sphere position sensor. The magnetic ball suspension system can be categorized into two systems: a mechanical system and an electrical system. The sphere position in the mechanical system can be controlled by adjusting the current through the electromagnet where the current through the electromagnet in the electrical system can be controlled by applying controlled voltage across the electromagnet terminals.

Usually, phenomenological models are nonlinear, that means at least one of the states ( $i_{\text {curren } t}, x_{\text {ball position }}$ ) is an argument of nonlinear function. In order to present such a model as a transfer function, the system has to be linearised. The simplest nonlinear model of the magnetic levitation system relating the ball position and the coil current $i$ is the following:

$$
m \ddot{x}=m g-F_{c}
$$

where $F_{c}=k \frac{i^{2}}{x^{2}}$ and $k$ is constant depending on the coil (electromagnet) parameters, $m g$ denotes the force due to the gravity while $F_{c}$ is the magnetic force generated by the coil. To present the full phenomenological model a relation between the control voltage $u$ and the coil current would have to be introduced analyzing the whole Maglev circuitry. However, Maglev is equipped with an inner control loop providing a current proportional to the control voltage that is generated for control purpose:

$$
i=k_{1} \cdot u
$$

Equation (1) \& (2) constitute a nonlinear model, which has been assembled in Simulink. The bound for the control signal is set to $[-5 \mathrm{~V}$ to $+5 \mathrm{~V}]$. To carry out analysis of the model dynamics for open loop system using techniques such as Bode plots, pole and zero maps, Nyquist plots, root locus (for closed loop system only), the model has to be linearised. Such a linearization is done in the equilibrium point of $x_{0}=$ $-1.5 \mathrm{~V}$ and $i_{0}=0.8 \mathrm{~A}$.

$$
\ddot{x}=g-f(x, i), \quad f(x, i)=k \frac{i^{2}}{m x^{2}}
$$

Equilibrium point can be calculated from:

$$
g=f(x, i) \Rightarrow i_{0}, x_{0}
$$

The linearization steps are following:

$$
\ddot{x}=-\left(\left.\frac{\partial f(i, x)}{\partial i}\right|_{i_{0} x_{0}} \Delta i+\left.\frac{\partial f(i, x)}{\partial x}\right|_{i_{0} x_{0}} \Delta x\right)
$$

Laplace transformation of equation (5) yields-

$$
s^{2} \Delta x=-\left(K_{i} \Delta i+K_{x} \Delta x\right)
$$

After simplification, equation (6) becomes

$$
\frac{\Delta x}{\Delta i}=\frac{-K_{i}}{s^{2}+K_{x}}
$$

where $K_{i}=\frac{2 m g}{i_{0}}$ and $K_{x}=-\frac{2 m g}{x_{0}}$

The metal sphere is sensed by an infra-red sensor which can discriminate the vertical movement of the ball from the horizontal ones. The minimum and the maximum distance of the metal sphere from the coil is $0.5 \mathrm{~cm}$ and $0.25 \mathrm{~cm}$, respectively.

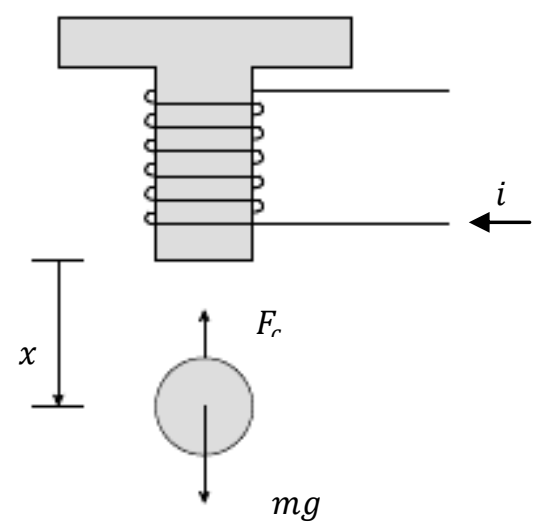

$\mathrm{T}$

Fig 2: Sketch of Magnetic Levitation System

$\dot{x}, u=$ (input command) and $y=k_{2} x$ (output in $\mathrm{cm}$ ).

$$
\left[\begin{array}{c}
\dot{x}_{1} \\
\dot{x}_{2} \\
y
\end{array}\right]=\left[\begin{array}{c}
x_{2} \\
g-\frac{k k_{1}^{2} u^{2}}{m x_{1}^{2}} \\
k_{2} x_{1}
\end{array}\right]
$$

\section{Table1. Parameters of Magnetic levitation system}

\begin{tabular}{|c|l|l|}
\hline $\boldsymbol{x}$ & Sphere Position & {$[0.005,0.025] \mathrm{m}$} \\
\hline $\boldsymbol{i}$ & Input current in the coil & {$[0,3] \mathrm{A}$} \\
\hline $\boldsymbol{u}$ & Input voltage & {$[0,5] \mathrm{V}$} \\
\hline $\boldsymbol{y}$ & Output sphere position & {$[0.5,2.5] \mathrm{cm}$} \\
\hline $\boldsymbol{m}$ & Mass of the metal sphere & $20 \times 10^{-3} \mathrm{Kg}$ \\
\hline $\boldsymbol{k}$ & Magnetic constant & $8.24 \times 10^{-5} \mathrm{Kg}$ \\
\hline $\boldsymbol{k}_{\mathbf{1}}$ & Input conductance & $0.3971 / \Omega$ \\
\hline $\boldsymbol{k}_{\mathbf{2}}$ & Unit conversion coefficient & $100 \mathrm{~cm} / \mathrm{m}$ \\
\hline $\boldsymbol{g}$ & Gravity acceleration & $9.8 \mathrm{~m} / \mathrm{s}^{2}$ \\
\hline
\end{tabular}




\section{CONTROLLER DESIGN}

In this section, control schemes like PID and Fuzzy logic controller are proposed

\subsection{PID Control}

The PID controller as shown schematically in Fig. 3, is well known and widely used to improve the dynamic response as well as to reduce or eliminate the steady state error. The derivative controller adds a finite zero to the open loop plant transfer function and improves the transient response. The integral controller adds a pole at the origin, thus increasing system type by one and reducing the steady state error due to a step function to zero.

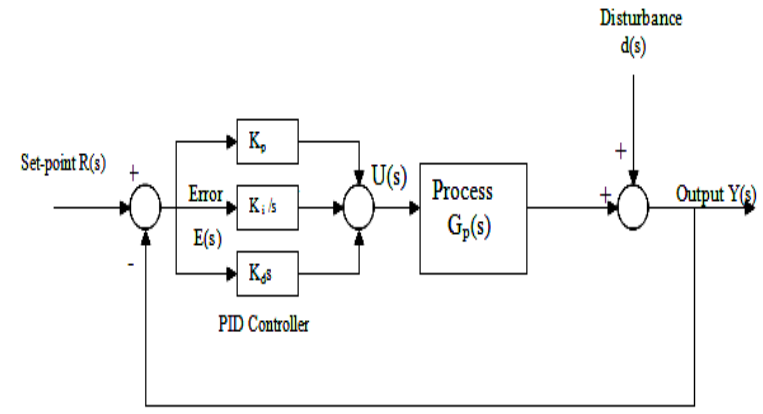

Fig 3: Schematic of conventional PID Controller

$$
U(s)=\left(K_{p}+\frac{K_{i}}{s}+s K_{d}\right) E(s)
$$

The parameters of PID controller are updated using ZeiglerNichols technique.

\subsection{Fuzzy Logic Controller}

The fuzzy sets concept was introduced by Zadeh in 1965 . The fuzzy algorithm can make human knowledge into the rule base to control a plant with linguistic descriptions. It relies on expert experience instead of mathematical models. The advantages of fuzzy control include good popularization, high faults tolerance, and suitable for nonlinear control systems. A fuzzy logic system (FLS) can be defined as the nonlinear mapping of an input data set to a scalar output data. A FLS consists of four main parts: fuzzifier, rules, inference engine, and defuzzifier. These components and the general architecture of a FLS are shown in Fig. 4.

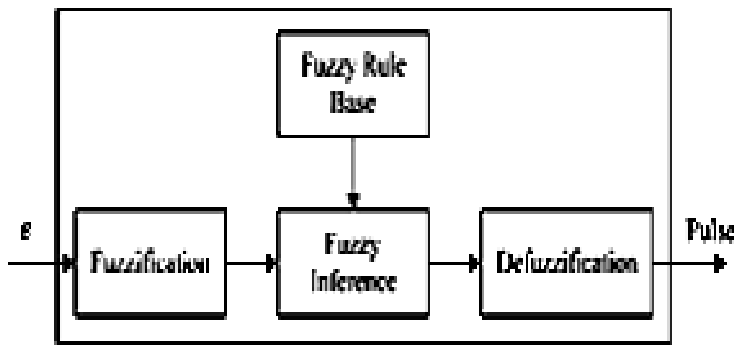

Fig 4: A Fuzzy Logic System

The fuzzy logic system is processed as: Firstly, crisp set of input data are gathered and converted to a fuzzy set using fuzzy linguistic variables, fuzzy linguistic terms and membership functions. This set is known as fuzzification. Afterwards, an inference is made based on a set of rules. Lastly, the resulting fuzzy output is mapped to a crisp output using the membership functions, in the defuzzification step. The evaluations of the fuzzy rules and the combination of the results of the individual rules are performed using fuzzy set operations. The operations on fuzzy sets are different than the operations on non-fuzzy sets. Let $\mu_{A}$ and $\mu_{B}$ are the membership functions for fuzzy sets $\mathrm{A}$ and $\mathrm{B}$. The mostlyused operations for OR and AND operators are max and min, respectively. For complement (NOT) operation, Eq. 10 is used for fuzzy sets.

$$
\mu_{\bar{A}}(x)=1-\mu_{A}(x)
$$

Fuzzy rules are expressed in the form of fuzzy conditional statements $R i$ of the type

\section{$R i$ : if $\mathbf{x}$ is small THEN $y$ is large}

Where $\mathrm{x}$ and $y$ are fuzzy variables, and small and large are labels of fuzzy set. If there are $\mathrm{i}=1$ to $\mathrm{n}$ rules, the rule set is represented by union of these rules

$$
R=R 1 \text { else } R 2 \text { else ...Rn }
$$

A fuzzy logic controller is based on a collection of $R$ control rules. The execution of these rules is governed by the compositional rule of inference.
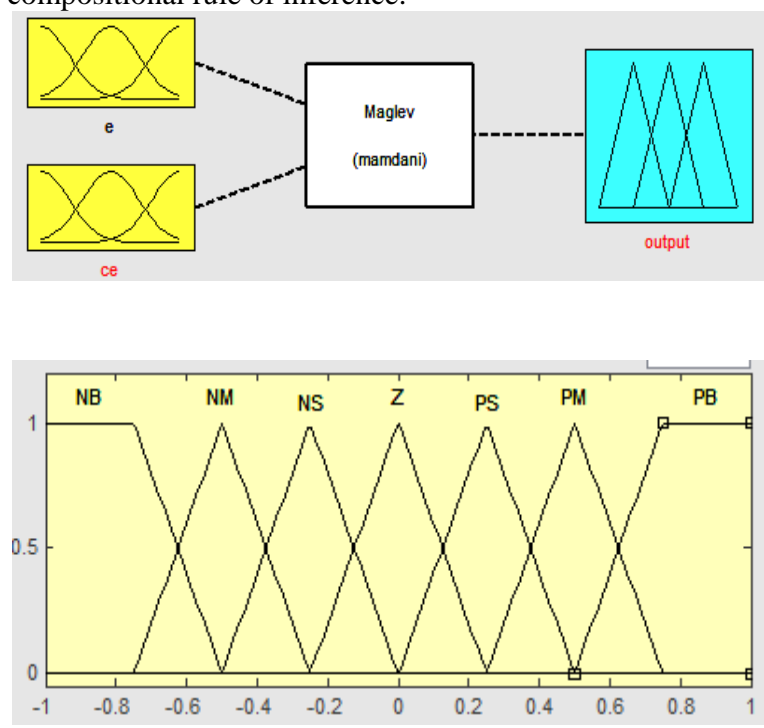

Fig 5: Input $e$ and ce Membership Function

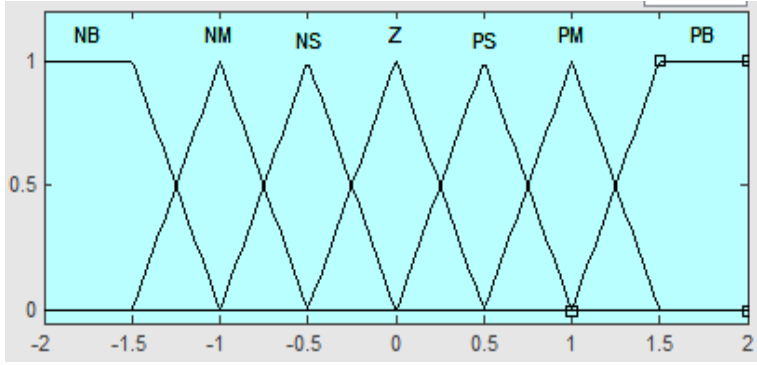

Fig 6: Output Membership Function

Table 2. Fuzzy Rule Base

\begin{tabular}{|c|c|c|c|c|c|c|c|}
\hline $\begin{array}{c}\text { ce } \\
\text { e }\end{array}$ & NB & NM & NS & Z & PS & PM & PB \\
\hline NB & NB & NB & NB & NB & NM & NS & Z \\
\hline NM & NB & NB & NB & NM & NS & Z & PS \\
\hline NS & NB & NB & NM & NS & Z & PS & PM \\
\hline
\end{tabular}




\begin{tabular}{|c|c|c|c|c|c|c|c|}
\hline$Z$ & NB & NM & NS & Z & PS & PM & PB \\
\hline PS & NM & NS & Z & PS & PM & PB & PB \\
\hline PM & NS & Z & PS & PM & PB & PB & PB \\
\hline PB & $Z$ & PS & PM & PB & PB & PB & PB \\
\hline
\end{tabular}

\section{RESULTS AND DISCUSSION}

The results shows that the controlled electromagnetic current can stabilize the disturbance that otherwise would cause the metal sphere to fall or attach itself to the electromagnet. In case of PID controller the integral action in magnetic levitation system can improve the system performance in terms of error minimization. However because of the fact that the system is not self starting the integral action has to be turned on when the ball acquires the stabilized position by the PD controller. Otherwise the huge error of ball position would be integrated by the controller resulting in unrealistic control values. This would cause system destabilization.

Maglev position control: $\mathrm{P}=4, \mathrm{D}=0.2$ and $\mathrm{I}=2$.

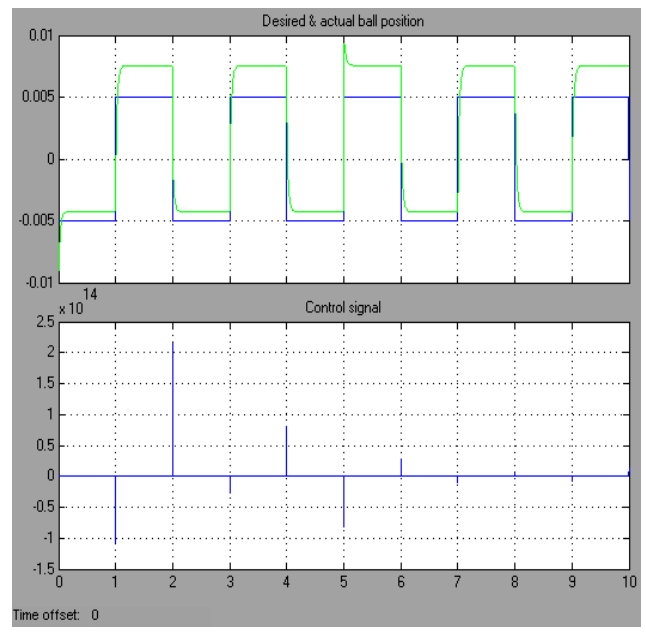

It can be noted that, FLC has a linear control surface. This is due to the equal widths of membership function for input and output. Fuzzy logic control has better tracking response as compared with the PID controller.

\section{CONCLUSION}

In this paper, the control strategies like PID and Fuzzy logic controller are successfully designed to control the magnetic levitation system. Based on the simulation results and also the experimental results, it is recorded that the fuzzy logic controller can stabilize the system efficiently and accurately more than PID controller.

\section{REFERENCES}

[1] K. Ishaque, S. S. Abdullah, S. Ayub, Z. Salam, "A simplified approach to design fuzzy logic controller for an underwater vehicle", Ocean Engineering, Vol. 38, No.1, 271-284 (2010).

[2] K.H.Ang, G.Chong, and Y.Li, "PID Control System Analysis, Design and Technology", IEEE Trans .on Control System Technology, vol.13, no.4, pp.559-576, 2005.

[3] S. Lei, A. Palazzolo, and A. Kascak. Fuzzy logic intelligent control system of magnetic bearings. In IEEE International Fuzzy Systems Conference, 2007. FUZZIEEE 2007, pages 1-6, 2007.

[4] T. Li, C. Kuo, and N. Guo. Design of an EP-based fuzzy sliding-mode control for a magnetic ball suspension system. Chaos, Solitons and Fractals, 33(5):1523-1531, 2007.

[5] Fuzzy Logic Toolbox For Use with MATLAB, The Mathworks Inc., version 2, Natick, MA, 2006.

[6] Cihan Karakuzu, Sitk1 öztürk ," A Comparison of Fuzzy, Neuro and Classical Control Techniques Based on an Experimental Application", University of Quafaquaz, No. 6, pp 189-198,July 2000.

[7] Meiyung Chen, Ming-Jyh Wang and Li-Chen Fu. "Modeling and controller design of a maglev guiding system for application in precision positioning, " IEEE Trans-actions on Industrial Electronics vol.50, no.3, pp.493-506, June 2003.

In case of Fuzzy logic control, the control strategy is based on human experience to construct the rule base for FLC.

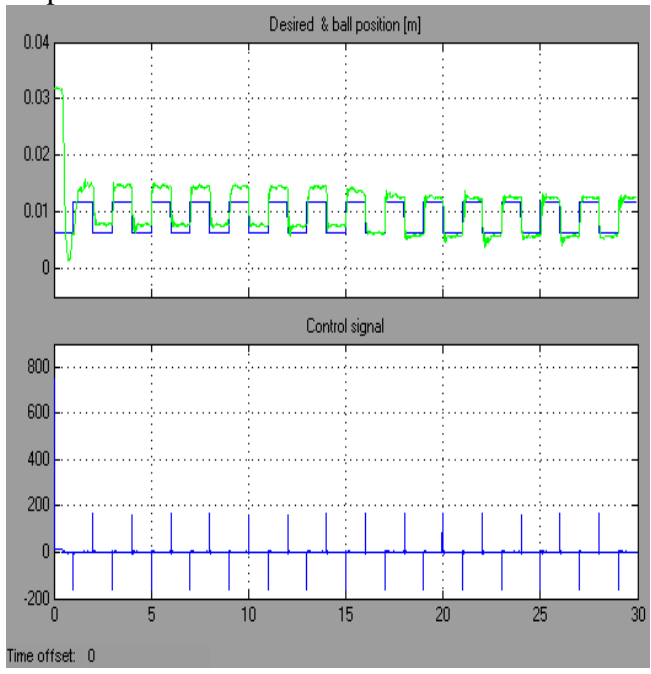

[8] G.K.I.Mann, B.G.Hu, and R.G.Gosine, "Analysis of Direct Action Fuzzy PID Controller structures", IEEE Trans. on System, Man and cybernetics, vol.29, no.3, pp.371-288,June 1999.

[9] R. C. Dorf and R. H. Bishop, Modern Control System, Addison Wesley, Menlo Park, CA, 1998, $8^{\text {th }}$ Ed.

[10] C. Lin and H. Jou. Force model identification for magnetic suspension systems via magnetic field measurement. IEEE Transactions on Instrumentation and Measurement, 42(3):767-771, 1993.

\section{AUTHORS PROFILE}

Shekhar Yadav was born in Bareilly, U.P, India in 1984. He received the degree of B.Tech from I.E.T.M.J.P.Rohilkhand University in 2007 and received the degree of M.Tech in Electrical Engineering from IT-BHU, Varanasi in 2010 and presently pursuing Ph.D in Electrical Engineering at IT-BHU, Varanasi, India. His research interests include control system, fuzzy logic system and optimization techniques.

J.P. Tewari was born in Deoria, U.P., India in 1947. He received the degrees of B.E. and M.E. from University of Roorkee, India in 1968 and 1971; and Ph.D in Electrical 
Engineering from Banaras Hindu University, India in 1992. $\mathrm{He}$ is currently Professor in Department of Electrical Engineering at IT-BHU, Varanasi, India. His main research includes Control systems, Robotics, Instrumentation, Control Systems, Adaptive control. He has published many papers in National \& International conferences and journals.

S.K.Nagar was born in Varanasi, India in 1955. He received the degrees of B.Tech and M.Tech from the Institute of Technology, Banaras Hindu University (IT-BHU), Varanasi,
India in 1976 and 1978; and Ph.D in Electrical Engineering from University of Roorkee (IIT Roorkee), India in 1991. He is currently Professor in Department of Electrical Engineering at IT-BHU, Varanasi, India. His main research includes digital control, discrete event systems and model order reduction. He has published many papers in National \& International conferences and journal 